\title{
Neutrosophic Soft Modules
}

Kemale Veliyeva, Sadi Bayramov

Department of Algebra and Geometry, of Baku State University, 23,, Z.Khalilov str.,, AZ1148, Baku, Azerbaijan

kemale2607@mail.ru, baysadi@gmail.com

\begin{abstract}
Molodtsov initiated the concept of soft sets in [17]. Maji et al. defined some operations on soft sets in [13]. Aktaş et al. generalized soft sets by defining the concept of soft groups in [2]. After then, QiuMei Sun et al. gave soft modules in [20]. In this paper, the concept of neutrosophic soft module is introduced and some of its basic properties are studied.
\end{abstract}

Key Words.. Neutrosophic set, neutrosophic soft set, neutrosophic soft modules, neutrosophic soft homomorphism.

Date of Publication: 30.5 .2018

DOI: $10.24297 / j a m . v 14 i 2.7401$

ISSN: 2347-1921

Volume: 14 Issue: 02

Journal: Journal of Advances in Mathematics

Website: https://cirworld.com

This work is licensed under a Creative Commons Attribution 4.0 International License.

\section{Introduction}

The contribution of mathematics to the present-day technology in reaching to a fast trend cannot be ignored. The treories presented differently from classical methods in studies such as fuzzy set [21],intuitionistic fuzzy set [3], soft set [17], neutrosophic set [19], etc. The algebraic structure of set theories dealing with uncertainties has also been studied by some authors.After Molodtsov's work, some different applications of soft sets were studied in [16]. Maji et al. [14] presented the concept of fuzzy soft set. Rosenfeld [18] proposed the concept of fuzzy groups in order to establish the algebraic structures of fuzzy sets. Aktaş and Çağman [2] defined soft groups and compared soft sets with fuzzy sets and rough sets. After the definition of fuzzy soft group is given by some authors [4,11]. F.Feng et al. [8] gave soft semirings and U.Acar et al. [1] introduced initial concepts of soft rings. Definition of fuzzy module is given by some authors [12,22]. Qiu- Mei Sun et al. [20] defined soft modules and investigated their basic properties. Fuzzy soft modules and intuitionistic fuzzy soft modules was given and researched by C. Gunduz (Aras) and S. Bayramov $[9,10]$.

The main purpose of this paper is to introduce a basic version of neutrosophic soft module theory, which extends the notion of module by including some algebraic structures in soft sets. Finally, we investigate some of neutrosophic soft module basic properties. 


\section{Preleminaries}

In this section, we will give some preliminary information for the present study.

Definition 2.1. [19] $A$ neutrosophic set $A$ on the universe of discourse $X$ is defined as:

$$
A=\left\{\left\langle x, \mathrm{~T}_{\mathrm{A}}(x), I_{\mathrm{A}}(x), F_{\mathrm{A}}(x)\right\rangle: x \in X\right\},
$$

where $\mathrm{T}, I, F: X \rightarrow]^{-} 0,1^{+}\left[\right.$and $-0 \leq \mathrm{T}_{\mathrm{A}}(x)+I_{\mathrm{A}}(x)+F_{\mathrm{A}}(x) \leq^{+} 3$.

Definition 2.2 [17] Let $X$ be an initial universe, $E$ be a set of all parameters and $P(X)$ denotes the power set of $X$.A pair $(F, E)$ is called a soft set over $X$, where $F$ is a mapping given by $F: E \rightarrow P(X)$.

Firstly, neutrosophic soft set defined by Maji [13] and later concept has been modified by Deli and Bromi [7] as given below:

Definition 2.3. Let $X$ be an initial universe set and $E$ be as a set of parameters. Let $P(X)$ denote the set of all neutrosophic sets of $X$. Then, a neutrosophic soft set $(\widetilde{F}, E)$ over $X$ is a set defined by a set valued function $\tilde{F}$ representing a mapping $\tilde{F}: E \rightarrow P(X)$ where $\tilde{F}$ is called approximate function of the neutrosophic soft set $(\tilde{F}, E)$. In other words, the neutrosophic soft set is a parameterized family of some elements of the set $P(X)$ and therefore it can be written as a set of ordered pairs,

$$
(\widetilde{F}, E)=\left\{\left(e,\left\langle x, \mathrm{~T}_{\widetilde{F}(e)}(x), I_{\widetilde{F}(e)}(x), F_{\widetilde{F}(e)}(x),\right\rangle: x \in X\right): e \in E\right\}
$$

where $\mathrm{T}_{\widetilde{F}(e)}(x), I_{\widetilde{F}(e)}(x), F_{\widetilde{F}(e)}(x) \in[0,1]$, respectively called the truth-membership, indeterminacymembership, falsity-membership function of $\tilde{F}(e)$. Since supremum of each T, $I, F$ is 1 so the inequality $0 \leq \mathrm{T}_{\widetilde{F}(e)}(x)+I_{\widetilde{F}(e)}(x)+F_{\widetilde{F}(e)}(x) \leq 3$ is obvious.

Definition 2.4. [6] Let $(\tilde{F}, E)$ be neutrosophic soft set over the common universe $(X, E)$. The complement of $(\tilde{F}, E)$ is denoted by $(\tilde{F}, E)^{c}$ and is defined by:

$$
(\tilde{F}, E)^{c}=\left\{\left(e,\left\langle x, F_{\widetilde{F}(e)}(x), 1-I_{\widetilde{F}(e)}(x), T_{\widetilde{F}(e)}(x),\right\rangle: x \in X\right): e \in E\right\} .
$$

Obvious that, $\left((\widetilde{F}, E)^{c}\right)^{c}=(\widetilde{F}, E)$.

Definition 2.5. [13] Let $(\tilde{F}, E)$ and $(\tilde{G}, E)$ be two neutrosophic soft sets over the common universe $(X, E) .(\widetilde{F}, E)$ is said to be neutrosophic soft subset of $(\widetilde{G}, E)$ if $\mathrm{T}_{\widetilde{F}(e)}(x) \leq \mathrm{T}_{\widetilde{G}(e)}(x), I_{\widetilde{F}(e)}(x) \leq I_{\widetilde{G}(e)}(x), F_{\widetilde{F}(e)}(x) \geq F_{\widetilde{G}(e)}(x), \quad \forall e \in E, \forall x \in X$. It is denoted by $(\tilde{F}, E) \subseteq(\widetilde{G}, E)$. 
The operations of union, intersection, difference, AND, OR on neutrosophic soft sets are defined differently from the studies [6,13]. In addition, basic properties of these operations will be presented.

Definition 2.6. Let $\left(\widetilde{F}_{1}, E\right)$ and $\left(\widetilde{F}_{2}, E\right)$ be two neutrosophic soft sets over the common universe $(X, E)$. Then their union is denoted by $\left(\widetilde{F}_{1}, E\right) \cup\left(\widetilde{F}_{2}, E\right)=\left(\widetilde{F}_{3}, E\right)$ and is defined by:

$$
\left(\widetilde{F}_{3}, E\right)=\left\{\left(e,\left\langle x, \mathrm{~T}_{\widetilde{F}_{3}(e)}(x), I_{\tilde{F}_{3}(e)}(x), F_{\widetilde{F}_{3}(e)}(x),\right\rangle: x \in X\right): e \in E\right\}
$$

where

$$
\begin{aligned}
& \mathrm{T}_{\tilde{F}_{3}(e)}(x)=\max \left\{\mathrm{T}_{\tilde{F}_{1}(e)}(x), \mathrm{T}_{\tilde{F}_{2}(e)}(x)\right\}, \\
& I_{\tilde{F}_{3}(e)}(x)=\max \left\{I_{\tilde{F}_{1}(e)}(x), I_{\tilde{F}_{2}(e)}(x)\right\}, \\
& F_{\tilde{F}_{3}(e)}(x)=\min \left\{F_{\tilde{F}_{1}(e)}(x) F_{\tilde{F}_{2}(e)}(x)\right\},
\end{aligned}
$$

Definition 2.7. Let $\left(\widetilde{F}_{1}, E\right)$ and $\left(\tilde{F}_{2}, E\right)$ be two neutrosophic soft sets over the common universe $(X, E)$. Then their union is denoted by $\left(\widetilde{F}_{1}, E\right) \cap\left(\widetilde{F}_{2}, E\right)=\left(\widetilde{F}_{3}, E\right)$ and is defined by:

$$
\left(\widetilde{F}_{3}, E\right)=\left\{\left(e,\left\langle x, \mathrm{~T}_{\widetilde{F}_{3}(e)}(x), I_{\widetilde{F}_{3}(e)}(x), F_{\widetilde{F}_{3}(e)}(x),\right\rangle: x \in X\right): e \in E\right\}
$$

where

$$
\begin{aligned}
& \mathrm{T}_{\tilde{F}_{3}(e)}(x)=\min \left\{\mathrm{T}_{\tilde{F}_{1}(e)}(x), \mathrm{T}_{\tilde{F}_{2}(e)}(x)\right\}, \\
& I_{\tilde{F}_{3}(e)}(x)=\min \left\{I_{\tilde{F}_{1}(e)}(x), I_{\tilde{F}_{2}(e)}(x)\right\}, \\
& F_{\tilde{F}_{3}(e)}(x)=\max \left\{F_{\tilde{F}_{1}(e)}(x) F_{\tilde{F}_{2}(e)}(x)\right\}
\end{aligned}
$$

Definition 2.8. Let $\left(\tilde{F}_{1}, E\right)$ and $\left(\tilde{F}_{2}, E\right)$ be two neutrosophic soft sets over the common universe $(X, E)$. Then " $\left(\widetilde{F}_{1}, E\right)$ difference $\left(\widetilde{F}_{2}, E\right)$ " operation on them is denoted by $\left(\tilde{F}_{1}, E\right) \backslash\left(\tilde{F}_{2}, E\right)=\left(\widetilde{F}_{3}, E\right)$ and is defined by $\left(\tilde{F}_{3}, E\right)=\left(\tilde{F}_{1}, E\right) \cap\left(\tilde{F}_{2}, E\right)^{c}$ as follows:

$$
\left(\widetilde{F}_{3}, E\right)=\left\{\left(e,\left\langle x, \mathrm{~T}_{\tilde{F}_{3}(e)}(x), I_{\tilde{F}_{3}(e)}(x), F_{\tilde{F}_{3}(e)}(x),\right): x \in X\right): e \in E\right\}
$$

where

$$
\begin{aligned}
& \mathrm{T}_{\tilde{F}_{3}(e)}(x)=\min \left\{\mathrm{T}_{\tilde{F}_{1}(e)}(x), F_{\tilde{F}_{2}(e)}(x)\right\}, \\
& I_{\tilde{F}_{3}(e)}(x)=\min \left\{I_{\tilde{F}_{1}(e)}(x), 1-I_{\widetilde{F}_{2}(e)}(x)\right\}, \\
& F_{\widetilde{F}_{3}(e)}(x)=\max \left\{F_{\widetilde{F}_{1}(e)}(x), T_{\widetilde{F}_{2}(e)}(x)\right\}
\end{aligned}
$$

Definition 2.9. Let $\left\{\left(\tilde{F}_{i}, E\right) \mid i \in I\right\}$ be a family of neutrosophic soft over the common universe $(X, E)$. Then 


$$
\begin{aligned}
& \bigcup_{i \in I}\left(\widetilde{F}_{i}, E\right)=\left\{\left(e,\left\langle x, \sup \left[\mathrm{T}_{\widetilde{F}_{i}(e)}(x)\right]_{i \in I}, \sup \left[I_{\widetilde{F}_{i}(e)}(x)\right]_{i \in I}, \inf \left[F_{\tilde{F}_{i}(e)}(x)\right]_{i \in I},\right\rangle: x \in X\right): e \in E\right\}, \\
& \bigcap_{i \in I}\left(\widetilde{F}_{i}, E\right)=\left\{\left(e,\left\langle x, \inf \left[\mathrm{T}_{\widetilde{F}_{i}(e)}(x)\right]_{i \in I}, \inf \left[I_{\tilde{F}_{i}(e)}(x)\right]_{i \in I}, \sup \left[F_{\widetilde{F}_{i}(e)}(x)\right]_{i \in I},\right\rangle: x \in X\right): e \in E\right\} .
\end{aligned}
$$

Definition 2.10. Let $\left(\tilde{F}_{1}, E\right)$ and $\left(\tilde{F}_{2}, E\right)$ be two neutrosophic soft sets over the common universe $(X, E)$. Then "AND" operation on them is denoted by $\left(\widetilde{F}_{1}, E\right) \wedge\left(\widetilde{F}_{2}, E\right)=\left(\widetilde{F}_{3}, E \times E\right)$ and is defined by:

$$
\left(\widetilde{F}_{3}, E \times E\right)=\left\{\left(\left(e_{1}, e_{2}\right),\left\langle x, \mathrm{~T}_{\widetilde{F}_{3}\left(e_{1}, e_{2}\right)}(x), I_{\widetilde{F}_{3}\left(e_{1}, e_{2}\right)}(x), F_{\widetilde{F}_{3}\left(e_{1}, e_{2}\right)}(x),\right\rangle: x \in X\right):\left(e_{1}, e_{2}\right) \in E \times E\right\}
$$

where

$$
\begin{aligned}
& \mathrm{T}_{\tilde{F}_{3}\left(e_{1}, e_{2}\right)}(x)=\min \left\{\mathrm{T}_{\tilde{F}_{1}\left(e_{1}\right)}(x), \mathrm{T}_{\tilde{F}_{2}\left(e_{2}\right)}(x)\right\}, \\
& I_{\tilde{F}_{3}\left(e_{1}, e_{2}\right)}(x)=\min \left\{I_{\tilde{F}_{1}\left(e_{1}\right)}(x), I_{\tilde{F}_{2}\left(e_{2}\right)}(x)\right\}, \\
& F_{\tilde{F}_{3}\left(e_{1}, e_{2}\right)}(x)=\max \left\{F_{\tilde{F}_{1}\left(e_{1}\right)}(x) F_{\tilde{F}_{2}\left(e_{2}\right)}(x)\right\} .
\end{aligned}
$$

Definition 2.11. Let $\left(\tilde{F}_{1}, E\right)$ and $\left(\tilde{F}_{2}, E\right)$ be two neutrosophic soft sets over the common universe $(X, E)$. Then "OR" operation on them is denoted by $\left(\widetilde{F}_{1}, E\right) \vee\left(\widetilde{F}_{2}, E\right)=\left(\widetilde{F}_{3}, E \times E\right)$ and is defined by:

$$
\left(\widetilde{F}_{3}, E \times E\right)=\left\{\left(\left(e_{1}, e_{2}\right),\left\langle x, \mathrm{~T}_{\widetilde{F}_{3}\left(e_{1}, e_{2}\right)}(x), I_{\widetilde{F}_{3}\left(e_{1}, e_{2}\right)}(x), F_{\widetilde{F}_{3}\left(e_{1}, e_{2}\right)}(x),\right\rangle: x \in X\right):\left(e_{1}, e_{2}\right) \in E \times E\right\}
$$

where

$$
\begin{aligned}
& \mathrm{T}_{\widetilde{F}_{3}\left(e_{1}, e_{2}\right)}(x)=\max \left\{\mathrm{T}_{\tilde{F}_{1}\left(e_{1}\right)}(x), \mathrm{T}_{\widetilde{F}_{2}\left(e_{2}\right)}(x)\right\}, \\
& I_{\widetilde{F}_{3}\left(e_{1}, e_{2}\right)}(x)=\max \left\{I_{\tilde{F}_{1}\left(e_{1}\right)}(x), I_{\tilde{F}_{2}\left(e_{2}\right)}(x)\right\}, \\
& F_{\tilde{F}_{3}\left(e_{1}, e_{2}\right)}(x)=\min \left\{F_{\tilde{F}_{1}\left(e_{1}\right)}(x) F_{\tilde{F}_{2}\left(e_{2}\right)}(x)\right\} .
\end{aligned}
$$

Definition 2.12. (1) $A$ neutrosophic soft set $(\tilde{F}, E)$ over $(X, E)$ is said to be null neutrosophic soft set if $\mathrm{T}_{\tilde{F}_{3}(e)}(x)=0, \quad I_{\tilde{F}_{3}(e)}(x)=0, \quad F_{\tilde{F}_{3}(e)}(x)=1 ; \quad \forall e \in E, \forall x \in X$. It is denoted by $0_{(X, E)}$.

(2) $A$ neutrosophic soft set $(\tilde{F}, E)$ over $(X, E)$ is said to be absolute neutrosophic soft set if $\mathrm{T}_{\widetilde{F}(e)}(x)=1, \quad I_{\widetilde{F}(e)}(x)=1, \quad F_{\widetilde{F}(e)}(x)=0 ; \quad \forall e \in E, \forall x \in X$. It is denoted by $1_{(X, E)}$.

Clearly $0_{(X, E)}^{c}=1_{(X, E)}$ and $1_{(X, E)}^{c}=0_{(X, E)}$.

Proposition 2.1. Let $\left(\widetilde{F}_{1}, E\right),\left(\widetilde{F}_{2}, E\right)$ and $\left(\widetilde{F}_{3}, E\right)$ be two neutrosophic soft sets over the common universe $(X, E)$. Then, 
(1) $\left(\tilde{F}_{1}, E\right) \cup\left[\left(\tilde{F}_{2}, E\right) \cup\left(\widetilde{F}_{3}, E\right)\right]=\left[\left(\tilde{F}_{1}, E\right) \cup\left(\tilde{F}_{2}, E\right)\right] \cup\left(\tilde{F}_{3}, E\right)$ and $\left(\tilde{F}_{1}, E\right) \cap\left[\left(\tilde{F}_{2}, E\right) \cap\left(\tilde{F}_{3}, E\right)\right]=\left[\left(\tilde{F}_{1}, E\right) \cap\left(\tilde{F}_{2}, E\right)\right] \cap\left(\tilde{F}_{3}, E\right) ;$

(2) $\quad\left(\tilde{F}_{1}, E\right) \cup\left[\left(\tilde{F}_{2}, E\right) \cap\left(\tilde{F}_{3}, E\right)\right]=\left[\left(\tilde{F}_{1}, E\right) \cup\left(\tilde{F}_{2}, E\right)\right] \cap\left[\left(\tilde{F}_{1}, E\right) \cup\left(\tilde{F}_{3}, E\right)\right]$ and $\left(\tilde{F}_{1}, E\right) \cap\left[\left(\tilde{F}_{2}, E\right) \cup\left(\tilde{F}_{3}, E\right)\right]=\left[\left(\tilde{F}_{1}, E\right) \cap\left(\tilde{F}_{2}, E\right)\right] \cup\left[\left(\tilde{F}_{1}, E\right) \cap\left(\widetilde{F}_{3}, E\right)\right] ;$

(3) $\left(\widetilde{F}_{1}, E\right) \cup 0_{(X, E)}=\left(\widetilde{F}_{1}, E\right)$ and $\left(\widetilde{F}_{1}, E\right) \cap 0_{(X, E)}=0_{(X, E)}$;

(4) $\left(\widetilde{F}_{1}, E\right) \cup 1_{(X, E)}=1_{(X, E)}$ and $\left(\widetilde{F}_{1}, E\right) \cap 1_{(X, E)}=\left(\widetilde{F}_{1}, E\right)$.

Proposition 2.2. Let $\left(\widetilde{F}_{1}, E\right)$ and $\left(\widetilde{F}_{2}, E\right)$ be two neutrosophic soft sets over the common universe $(X, E)$. Then,

(1) $\quad\left[\left(\widetilde{F}_{1}, E\right) \cup\left(\widetilde{F}_{2}, E\right)\right]^{c}=\left(\widetilde{F}_{1}, E\right)^{c} \cap\left(\widetilde{F}_{2}, E\right)^{c}$;

(2) $\quad\left[\left(\widetilde{F}_{1}, E\right) \cap\left(\widetilde{F}_{2}, E\right)\right]^{c}=\left(\widetilde{F}_{1}, E\right)^{c} \cup\left(\widetilde{F}_{2}, E\right)^{c}$.

Proposition 2.3. Let $\left(\tilde{F}_{1}, E\right)$ and $\left(\widetilde{F}_{2}, E\right)$ be two neutrosophic soft sets over the common universe $(X, E)$. Then,

(1) $\left[\left(\widetilde{F}_{1}, E\right) \vee\left(\widetilde{F}_{2}, E\right)\right]^{c}=\left(\widetilde{F}_{1}, E\right)^{c} \wedge\left(\widetilde{F}_{2}, E\right)^{c}$;

(2) $\left[\left(\tilde{F}_{1}, E\right) \wedge\left(\tilde{F}_{2}, E\right)\right]^{c}=\left(\tilde{F}_{1}, E\right)^{c} \vee\left(\tilde{F}_{2}, E\right)^{c}$.

Definition 2.13. Let $\mathrm{M}$ be a left $R$-module and let $A=(T, I, F)$ be a neutrosophic set over M. Then we say $(M, T, I, F)$ is a neutrosophic modul, if the following conditions are satisfied:
a) $T(0)=I(0)=1 ; \quad F(0)=0$
b) $T(x+y) \geq T(x) \wedge T(y) ; I(x+y) \geq I(x) \wedge I(y) ; F(x+y) \leq \max \{F(x), F(y)\}$
c) $T(\lambda x) \geq T(x) ; I(\lambda x) \geq I(x) ; F(\lambda x) \leq F(x)$

Definition 2.14. Let $\left(M_{1}, T_{1}, I_{1}, F_{1}\right)$ and $\left(M_{2}, T_{2}, I_{2}, F_{2}\right)$ be two neutrosophic modules over $M_{1}$ and $M_{2}$, respectively. We say that $f$ is a homomorphism of neutrosophic modules, if the following conditions for homomorphism of $f: M_{1} \rightarrow M_{2}$ modules are satisfied:

$$
T_{2}(f(x)) \geq T_{1}(x), I_{2}(f(x)) \geq I_{1}(x), F_{2}(f(x)) \leq F_{1}(x)
$$

\section{Neutrosophic soft modules}

In this paper $R$ is an ordinary ring. Let $M$ be a left (or right) $R$-module and let $A \neq \varnothing$ be a set. $N S(M)$ denotes the family of neutrosophic sets over $M$.

Definition 3.1. Let $(\tilde{F}, A)$ be a neutrosophic soft set over $M$. Then $(\tilde{F}, A)$ is said to be a neutrosophic soft module over $M$ iff $\forall a \in A, \tilde{F}(a)=\left(T_{a}, I_{a}, F_{a}\right)$ is a neutrosophic submodule of $M$ and denoted as $\tilde{F}_{a}$. 
Definition 3.2. Let $\left(\tilde{F}^{1}, A\right)$ and $\left(\tilde{F}^{2}, B\right)$ be two neutrosophic soft modules over $M$ and $N$ respectively, and let $f: M \rightarrow N$ be a homomorphism of modules, and let $g: A \rightarrow B$ be a mapping of sets. Then we say that $(f, g):\left(\tilde{F}^{1}, A\right) \rightarrow\left(\tilde{F}^{2}, B\right)$ is a neutrosophic soft homomorphism of neutrosophic soft modules, if the following condition is satisfied:

$$
f\left(T_{(a)}^{1}\right)=\tilde{F}^{2}(g(a))=T_{g(a)}^{2}, f\left(I_{(a)}^{1}\right)=\tilde{F}^{2}(g(a))=I_{g(a)}^{2}, f\left(F_{(a)}^{1}\right)=\tilde{F}^{2}(g(a))=F_{g(a)}^{2}
$$

Note that for $\forall a \in A, \quad f:\left(M, \tilde{F}_{(a)}^{1}\right) \rightarrow\left(N, \tilde{F}_{g(a)}^{2}\right)$ is a neutrosophic homomorphism of neutrosophic modules.

Neutrosophic soft modules and morphisms of their is consists of a category. This category is denoted NSM .

Theorem 3.1. Let $\left(\tilde{F}^{1}, A\right)$ and $\left(\tilde{F}^{2}, B\right)$ be two neutrosophic soft modules over $M$. Then their intersection $\left(\tilde{F}^{1}, A\right) \cap\left(\tilde{F}^{2}, B\right)$ is a neutrosophic soft module over $M$.

Proof. Let $\left(\tilde{F}^{1}, A\right) \cap\left(\tilde{F}^{2}, B\right)=\left(\tilde{F}^{3}, C\right)$, where $C=A \cap B$. Since the neutrosophic soft set $T_{c}^{3}=T_{c}^{1} \wedge T_{c}^{2}, I_{c}^{3}=I_{c}^{1} \wedge I_{c}^{2}, F_{c}^{3}=F_{c}^{1} \vee F_{c}^{2}$ is a neutrosophic submodule, for $\forall c \in C,\left(\tilde{F}^{3}, C\right)$ is a neutrosophic soft module over $M$.

Theorem 3.2. Let $\left(\tilde{F}^{1}, A\right)$ and $\left(\tilde{F}^{2}, B\right)$ be two neutrosophic soft modules over $M$. Then $\left(\tilde{F}^{1}, A\right) \wedge\left(\widetilde{F}^{2}, B\right)$ is a neutrosophic soft module over $M$.

Proof. We can write $\left(\tilde{F}^{1}, A\right) \wedge\left(\tilde{F}^{2}, B\right)=\left(\tilde{F}^{3}, A \times B\right)$. Since $\tilde{F}_{a}^{1}$ and $\tilde{F}_{b}^{2}$ are neutrosophic submodules of $M, \quad \tilde{F}_{a}^{1} \wedge \tilde{F}_{b}^{2}$ is a neutrosophic submodule of $M$. Thus, $T^{3}(a, b)=T_{a}^{1} \wedge T_{b}^{2}, I^{3}(a, b)=I_{a}^{1} \wedge I_{b}^{2}, F^{3}(a, b)=F_{a}^{1} \vee F_{b}^{2}$ is a neutrosophic submodule of $M$ ， for all $(a, b) \in A \times B$. Hence, we find that $\left(\widetilde{F}^{1}, A\right) \wedge\left(\widetilde{F}^{2}, B\right)$ is a neutrosophic soft module over $M$ .Theorem 3.3. Let $\left(\tilde{F}^{1}, A\right)$ and $\left(\tilde{F}^{2}, B\right)$ be two neutrosophic soft modules over $M$. If $A \cap B=\varnothing$, then $\left(\widetilde{F}^{1}, A\right) \cup\left(\tilde{F}^{2}, B\right)$ is a neutrosophic soft module over $M$.

Proof. We can write $\left(\tilde{F}^{1}, A\right) \cup\left(\tilde{F}^{2}, B\right)=\left(\tilde{F}^{3}, C\right)$. Since $A \cap B=\varnothing$, it follows that either $c \in A-B$ or $c \in B-A$ for all $c \in C$. If $c \in A-B$, then $\tilde{F}_{b}^{3}=\tilde{F}_{b}^{1}$ is a neutrosophic submodule of $M$, and if $c \in B-A$, then $\tilde{F}_{b}^{3}=\tilde{F}_{b}^{2}$ is a neutrosophic submodule of $M$. Hence, $\left(\tilde{F}^{1}, A\right) \cup\left(\tilde{F}^{2}, B\right)$ is a neutrosophic soft module over $M$.

Definition 3.3. Let $\left(\tilde{F}^{1}, A\right)$ and $\left(\tilde{F}^{2}, B\right)$ be two neutrosophic soft modules over $M$. Then $\left(\tilde{F}^{1}, A\right)$ is called a neutrosophic soft submodule of $\left(\tilde{F}^{2}, B\right)$ if

1) $A \subset B$

2) For all $a \in A, \tilde{F}_{a}^{1}=\left(T_{a}^{1}, I_{a}^{1}, F_{a}^{1}\right)$ is a neutrosophic submodule of $\tilde{F}_{a}^{2}=\left(T_{a}^{2}, I_{a}^{2}, F_{a}^{2}\right)$, $T_{a}^{1} \leq T_{a}^{2}, I_{a}^{1} \leq I_{a}^{2}, F_{a}^{1} \geq F_{a}^{2}$. 
Theorem 3.4. Let $\left(\tilde{F}^{1}, A\right)$ and $\left(\tilde{F}^{2}, A\right)$ be two neutrosophic soft modules over $M$. If $\tilde{F}_{a}^{1} \leq \tilde{F}_{a}^{2}$ for all $a \in A$, then $\left(\widetilde{F}^{1}, A\right)$ is a neutrosophic soft submodule of $\left(\tilde{F}^{2}, A\right)$.

Proof. The proof of the theorem is straightforward.

Theorem 3.5. Let $(\tilde{F}, A)$ be a neutrosophic soft modules over $M$, and let $\left\{\left(\tilde{F}_{i}, A_{i}\right)\right\}_{i \in I}$ be nonempty family of neutrosophic soft submodules of $(\tilde{F}, A)$. Then

1) $\prod_{i \in I}\left(\tilde{F}_{i}, A_{i}\right)$ is a neutrosophic soft submodule of $(\tilde{F}, A)$,

2) $\wedge_{i \in I}\left(\widetilde{F}_{i}, A_{i}\right)$ is a neutrosophic soft submodule of $(\tilde{F}, A)$,

3) If $A_{i} \cap A_{j}=\varnothing$, for all $i, j \in I$, then $\underset{i \in I}{\vee}\left(\tilde{F}_{i}, A_{i}\right)$ is a neutrosophic soft submodule of $(\tilde{F}, A)$.

Let $\left(\tilde{F}^{1}, A\right)$ and $\left(\tilde{F}^{2}, B\right)$ be two neutrosophic soft modules over $M$ and $N$ respectively, and $(f, g):\left(\tilde{F}^{1}, A\right) \rightarrow\left(\tilde{F}^{2}, B\right)$ be a neutrosophic soft homomorphism of these modules.

Now in this section, we introduce the kernel and image of neutrosophic soft homomorphism of neutrosophic soft modules. Let $M^{\prime}=\operatorname{ker} f$. Define $\tilde{F}^{\prime}: A \rightarrow N S\left(M^{\prime}\right)$ by $T_{a}^{\prime}=\left.T_{a}\right|_{M^{\prime}}, I_{a}^{\prime}=\left.I_{a}\right|_{M^{\prime}}, F_{a}^{\prime}=\left.F_{a}\right|_{M^{\prime}}$. Then $\left(\tilde{F}^{\prime}, A\right)$ is a neutrosophic soft module over $M^{\prime}$. It is clear that this module is a neutrosophic soft submodule of $(\tilde{F}, A)$.

Definition 3.4. $\left(\tilde{F}^{\prime}, A\right)$ is said to be kernel of $(f, g)$ and denoted by $\operatorname{ker}(f, g)$.

Now, let $B^{\prime}=g(A)$. Then for all $b \in B^{\prime}$, there exists $a \in A$ such that $g(a)=b$. Let $N^{\prime}=\operatorname{Im} f<N$. We define the mapping $\tilde{F}^{\prime 2}: B^{\prime} \rightarrow N S\left(N^{\prime}\right)$ as $T^{\prime 2}\left(b^{\prime}\right)=\left.T^{2}(g(a))\right|_{N^{\prime}}, I^{\prime 2}\left(b^{\prime}\right)=I^{2}(g(a))_{N^{\prime}}, F^{\prime 2}\left(b^{\prime}\right)=\left.F^{2}(g(a))\right|_{N^{\prime}}$. Since $\quad(f, g) \quad$ is a neutrosophic soft homomorphism, $f\left(T_{a}^{1}\right)=T_{g(a)}^{2}, f\left(I_{a}^{1}\right)=I_{g(a)}^{2}, f\left(F_{a}^{1}\right)=F_{g(a)}^{2}$ is satisfied for all $a \in A$. Then the pair $\left(\tilde{F}^{\prime 2}, B^{\prime}\right)$ is a neutrosophic soft module over $N^{\prime}$ and $\left(\tilde{F}^{\prime 2}, B^{\prime}\right)$ is a neutrosophic soft submodule of $\left(\tilde{F}^{2}, B\right)$.

Definition 3.5. $\left(\tilde{F}^{\prime 2}, B^{\prime}\right)$ is said to be image of $(f, g)$ and denoted by $\operatorname{Im}(f, g)$.

Proposition 3.1. Let $(\tilde{F}, A)$. be a neutrosophic soft module over $M, N$ be an $R$-module and $f: M \rightarrow N$. be a homomorphism of $R$-modules. Then $(f(\tilde{F}), A)$. is a neutrosophic soft module over $N$.

Proof. If the mapping $f(\tilde{F}): A \rightarrow N S(N)$ is defined by 


$$
\begin{aligned}
& (f(T))_{a}(y)=\sup \left\{T_{a}(x): f(x)=y\right\} \\
& (f(I))_{a}(y)=\sup \left\{I_{a}(x): f(x)=y\right\} \\
& (f(F))_{a}(y)=\inf \left\{F_{a}(x): f(x)=y\right\}
\end{aligned}
$$

the proof is completed.

Note that $\left(f, 1_{A}\right):(\tilde{F}, A) \rightarrow(f(\tilde{F}), A)$ is a neutrosophic soft homomorphism of neutrosophic soft modules.

Proposition 3.2. If $M$ is an $R$-module, $(\tilde{F}, A)$ is a neutrosophic soft module over $N$ and $f: M \rightarrow N$ is a homomorphism of $R$-modules, then $\left(f^{-1}(\tilde{F}), A\right)$ is a neutrosophic soft module over $M$.

Proof. If the mapping $f^{-1}(\tilde{F}): A \rightarrow N S(M)$ is defined by

$$
\left(f^{-1}(T)\right)_{a}(x)=T_{a}(f(x)),\left(f^{-1}(I)\right)_{a}(x)=I_{a}(f(x)),\left(f^{-1}(F)\right)_{a}(x)=F_{a}(f(x)),
$$

the proof is completed.

It is clear that $\left(f, 1_{A}\right):\left(f^{-1}(\tilde{F}), A\right) \rightarrow(\widetilde{F}, A)$ is a neutrosophic soft homomorphism of neutrosophic soft modules.

Lemma 3.1. Let $M$ and $N$ be an $R$-modules and $f: M \rightarrow N$ be an $R$-homomorphism and $\left(\tilde{F}^{1}, A\right)$ and $\left(\tilde{F}^{2}, A\right)$ are two neutrosophic soft modules over $M$ and $N$ respectively.

(i) $\left(f, 1_{A}\right):\left(\widetilde{F}^{1}, A\right) \rightarrow\left(\tilde{F}^{2}, A\right)$ is a neutrosophic soft homomorphism if and only if for all $a \in A$, $T_{a}^{2} \geq f\left(T_{a}^{1}\right), I_{a}^{2} \geq f\left(I_{a}^{1}\right), F_{a}^{2} \leq f\left(F_{a}^{1}\right)$ is satisfied.

(ii) $\left(f, 1_{A}\right):\left(\tilde{F}^{1}, A\right) \rightarrow\left(\tilde{F}^{2}, A\right)$ is a neutrosophic soft homomorphism if and only if for all $a \in A$, $T_{a}^{1} \leq f^{-1}\left(T_{a}^{2}\right), I_{a}^{1} \leq f^{-1}\left(I_{a}^{2}\right), F_{a}^{1} \geq f^{-1}\left(F_{a}^{2}\right)$ is satisfied.

Theorem 3.6. If $\left\{\left(\tilde{F}_{i}, A_{i}\right)\right\}_{i \in I}$ is a family of neutrosophic soft modules over $\left\{M_{i}\right\}_{i \in I}$, then $\prod_{i \in I}\left(\tilde{F}_{i}, A_{i}\right)$ is a neutrosophic soft module over $\prod_{i \in I} M_{i}$.

Proof. Define $\tilde{F}: \prod_{i \in I} A_{i} \rightarrow \prod_{i \in I} M_{i} \tilde{F}=(T, I, F)$ by

$$
T\left(\left\{a_{i}\right\}\right)=\underset{i \in I}{\vee} p_{i}^{-1}\left(T_{i}\right)_{a_{i}}, I\left(\left\{a_{i}\right\}\right)=\underset{i \in I}{\vee} p_{i}^{-1}\left(I_{i}\right)_{a_{i}}, F\left(\left\{a_{i}\right\}\right)=\underset{i \in I}{\wedge} p_{i}^{-1}\left(F_{i}\right)_{a_{i}}
$$

where $p_{i}: \prod_{i \in I} M_{i} \rightarrow M_{i}$ is a projection mapping. Since

$p_{i}^{-1}\left(T_{i}\right)_{a_{i}}: \prod_{i \in I} M_{i} \rightarrow[0,1], p_{i}^{-1}\left(I_{i}\right)_{a_{i}}: \prod_{i \in I} M_{i} \rightarrow[0,1], p_{i}^{-1}\left(F_{i}\right)_{a_{i}}: \prod_{i \in I} M_{i} \rightarrow[0,1]$ 
is a neutrosophic soft module over $\prod_{i \in I} M_{i}$, for all $i \in I, \underset{i \in I}{\vee} p_{i}^{-1}\left(T_{i}\right)_{a_{i}}, \underset{i \in I}{\vee} p_{i}^{-1}\left(I_{i}\right)_{a_{i}}, \wedge \underset{i \in I}{\wedge} p_{i}^{-1}\left(F_{i}\right)_{a_{i}}$ is also a neutrosophic soft module over $\prod_{i \in I} M_{i}$.

Theorem 3.7. If $\left\{\left(\tilde{F}_{i}, A_{i}\right)\right\}_{i \in I}$ is a family of neutrosophic soft modules over the family of modules $\left\{M_{i}\right\}_{i \in I}$, then $\underset{i \in I}{\oplus}\left(\tilde{F}_{i}, A_{i}\right)$ is a neutrosophic soft module over $\underset{i \in I}{\oplus} M_{i}$.

Proof. Define $\quad \tilde{F}: \prod_{i \in I} A_{i} \rightarrow \bigoplus_{i \in I} M_{i} \quad \tilde{F}=(T, I, F) \quad$ for $\quad$ all $\quad\left\{a_{i}\right\} \in \prod_{i \in I} A_{i} \quad$ by $T\left(\left\{a_{i}\right\}\right)=\underset{i \in I}{\wedge} j_{i}\left(T_{i}\right)_{a_{i}}, I\left(\left\{a_{i}\right\}\right)=\underset{i \in I}{\wedge} j_{i}\left(I_{i}\right)_{a_{i}}, F\left(\left\{a_{i}\right\}\right)=\underset{i \in I}{\vee} j_{i}\left(F_{i}\right)_{a_{i}} \quad$ where $\quad j_{i}: M_{i} \rightarrow \underset{i \in I}{\oplus} M_{i} \quad$ is a embedding mapping. Since $j_{i}\left(T_{i}\right)_{a_{i}}, j_{i}\left(I_{i}\right)_{a_{i}}, j_{i}\left(F_{i}\right)_{a_{i}}$ is a neutrosophic soft submodule over $\underset{i \in I}{\oplus} M_{i}$ for all $i \in I, F\left(\left\{a_{i}\right\}\right)$ is a neutrosophic submodule over $\underset{i \in I}{\oplus} M_{i}$.

Lemma 3.2. 1) Given modules $\left\{M_{i}\right\}_{i \in I}$ and $N$ and a family of $R$-homorphisms $A=\left\{f_{i}: M_{i} \rightarrow N\right\}_{i \in I}$. If $\left\{\left(\widetilde{F}_{i}, A_{i}\right)\right\}_{i \in I}$ are neutrosophic soft modules over $\left\{M_{i}\right\}_{i \in I}$, then there exist a neutrosophic soft module $\left(\tilde{F}^{2}, \prod_{i \in I} A_{i}\right)$ over $N$ such that for all $i \in I$,

$$
f_{i}:\left(\tilde{F}_{i}, A_{i}\right) \rightarrow\left(\tilde{F}^{2}, \prod_{i \in I} A_{i}\right)
$$

is a neutrosophic soft homomorphism of neutrosophic soft modules.

2) Given modules $M$ and $\left\{N_{i}\right\}_{i \in I}$ and a family of $R$-homorphisms $B=\left\{g_{i}: M \rightarrow N_{i}\right\}_{i \in I}$. If $\left\{\left(\tilde{F}_{i}^{2}, B_{i}\right)\right\}_{i \in I}$ are neutrosophic soft modules over $\left\{N_{i}\right\}_{i \in I}$, then there exist a neutrosophic soft module $\left(\tilde{F}, \prod_{i \in I} A_{i}\right)$ over $M$ such that for all $i \in I$,

$$
g_{i}:\left(\tilde{F}, \prod_{i \in I} A_{i}\right) \rightarrow\left(\tilde{F}_{i}^{2}, B_{i}\right)
$$

is a neutrosophic soft homomorphism of neutrosophic soft modules.

Proof. 1) Define $\tilde{F}^{2}: \prod_{i \in I} A_{i} \rightarrow N, \tilde{F}^{2}=\left(T^{2}, I^{2}, F^{2}\right)$ by $T^{2}\left(\left\{a_{i}\right\}\right)=\underset{i}{\vee} f_{i}\left(T_{i}^{2}\right)_{a_{i}}, I^{2}\left(\left\{a_{i}\right\}\right)=\underset{i}{\vee} f_{i}\left(I_{i}^{2}\right)_{a_{i}}, F^{2}\left(\left\{a_{i}\right\}\right)=\underset{i}{\wedge} f_{i}\left(F_{i}^{2}\right)_{a_{i}}$

2) Define $\tilde{F}: \prod_{i \in I} A_{i} \rightarrow M, \tilde{F}=(T, I, F)$ by $T\left(\left\{a_{i}\right\}\right)=\hat{i \in I}_{i \in I} g_{i}^{-1}\left(T_{i}\right)_{a_{i}}, I\left(\left\{a_{i}\right\}\right)=\hat{i \in I}_{i \in I} g_{i}^{-1}\left(I_{i}\right)_{a_{i}}, F\left(\left\{a_{i}\right\}\right)={\underset{i \in I}{\vee}}_{g_{i}^{-1}}\left(F_{i}\right)_{a_{i}}$ for all $\left\{a_{i}\right\} \in \prod_{i \in I} A_{i}$. 
By using this lemma, we define the concepts of submodule, quotient module, product and coproduct operations in the category of neutrosophic soft modules.

Corallary 3.1. If $(\tilde{F}, A)$ is a neutrosophic soft module over $M$ and $N$ is a submodule of $M$ and $i: N \rightarrow M$ is a embedding mapping, then $\left(i^{-1}(\widetilde{F}), A\right)$ is a neutrosophic soft module over $N$.

Corallary 3.2. If $(\tilde{F}, A)$ is a neutrosophic soft module over $M$ and $p: M \rightarrow M / \sim$ is a canonical projection, then $(p(\tilde{F}), A)$ is a neutrosophic soft module over quotient module $M / \sim$.

If $\left\{\left(\tilde{F}_{i}, A_{i}\right)\right\}_{i \in I}$ is a family of neutrosophic soft modules over the family of modules $\left\{M_{i}\right\}_{i \in I}$, then we can define the product and coproduct of these families by $\prod_{i \in I}\left(\tilde{F}_{i}, A_{i}\right)$ and $\underset{i \in I}{\oplus}\left(\tilde{F}_{i}, A_{i}\right)$, respectively.

Theorem 3.8. The category of neutrosophic soft modules has zero objects, sums, product, kernel and cokernel.

Let $M$ and $N$ be, respectively, right and left modules over $R$ (ring). Let $\left(\tilde{F}^{1}, A\right)$ and $\left(\tilde{F}^{2}, B\right)$ be two neutrosophic soft modules over $M$ and $N$, respectively. We consider tensor product of modules as $M \otimes N$. The mapping

$$
\tilde{F}^{1} \otimes \tilde{F}^{2}: A \times B \rightarrow M \otimes N
$$

is defined by

$$
\begin{aligned}
& \left(T^{1} \otimes T^{2}\right)(a, b)=T^{1}(a) \otimes T^{2}(b), \\
& \left(I^{1} \otimes I^{2}\right)(a, b)=I^{1}(a) \otimes I^{2}(b), \\
& \left(F^{1} \otimes F^{2}\right)(a, b)=F^{1}(a) \otimes F^{2}(b)
\end{aligned}
$$

for $\forall(a, b) \in A \times B$.

Definition 3.6. $\left(\widetilde{F}^{1} \otimes \tilde{F}^{2}, A \times B\right)$ is said to be tensor product of $\left(\tilde{F}^{1}, A\right)$ and $\left(\tilde{F}^{2}, B\right)$ and denoted by $\left(\tilde{F}^{1}, A\right) \otimes\left(\tilde{F}^{2}, B\right)$.

Theorem 3.9. $\left(\widetilde{F}^{1} \otimes \widetilde{F}^{2}, A \times B\right)$ is a neutrosophic soft module over $M \otimes N$.

Proof. For $\forall(a, b) \in A \times B,\left(M, \tilde{F}_{a}^{1}\right)$ and $\left(N, \tilde{F}_{b}^{2}\right)$ are neutrosophic soft modules. $\tilde{F}_{a}^{1} \otimes \widetilde{F}_{b}^{2}$ is a neutrosophic submodule over $M \otimes N$ and $\left(\widetilde{F}^{1} \otimes \tilde{F}^{2}, A \times B\right)$ is a neutrosophic soft module over $M \otimes N$.

Definition 3.7. $\left(\tilde{F}^{1} \otimes \tilde{F}^{2}, A \times B\right)$ is said to be tensor product of $\left(\tilde{F}^{1}, A\right)$ and $\left(\tilde{F}^{2}, B\right)$, and denoted by $\left(\widetilde{F}^{1}, A\right) \otimes\left(\tilde{F}^{2}, B\right)$. 


\section{Conclusion}

This paper summarized the basic concepts of neutrosophic soft sets and neutrosophic soft modules. By using these concepts, we studied the algebraic properties of neutrosophic soft sets in module structure.

\section{Reference}

1. U. Acar, F.Koyuncu, B.Tanay, Soft sets and soft rings, Comput. Math. Appl.59 (2010) 3458-3463.

2. H.Aktaş, N. Çağman, Soft sets and soft group, Information Science 177 (2007) 2726-2735.

3. Atanassov K., Intuitionistic fuzzy sets, Fuzzy Sets and Systems 20 (1986) 87-96.

4. A.Aygünoğlu, H.Aygün, Introduction to fuzzy soft groups, Comput. Math. Appl.58 (2009) 12791286.

5. Bera T., Mahapatra N. K., On neutrosophic soft function, Ann. Fuzzy Math. Inform. 12(1), (2016) 101-119.

6. Bera T., Mahapatra N. K., Introduction to neutrosophic soft topological space, Opsearch, 54(4), (2017) 841-867.

7. Deli I., Broumi S., Neutrosophic soft relations and some properties, Ann. Fuzzy Math. Inform. 9(1), (2015) 169-182.

8. F. Feng, Y.B. Jun, X. Zhao, Soft semirings, Comput. Math. Appl.56 (2008) 2621-2628.

9. C.Gunduz (Aras) and S. Bayramov, Fuzzy soft modules, International Mathematical Forum, Vol. 6, 2011, no.11, 517-527.

10. C.Gunduz (Aras) and S. Bayramov, Intuitionistic fuzzy soft modules, Computer and Mathematics with Application, 62(2011), 2480-2486.

11. L.Jin-liang, Y.Rui-xia, Y.Bing-xue, Fuzzy soft sets anf fuzzy soft groups, Chinese Control and Decision Conference (2008), 2626-2629.

12. S.R.Lopez-Permouth, D.S.Malik, On categories of fuzzy modules, Information Sciences 52 (1990) 211-220.

13. Maji P. K., Neutrosophic soft set, Ann. Fuzzy Math. Inform. 5(1), (2013) 157-168.

14. P.K.Maji, R.Bismas, A.R.Roy, Fuzzy soft sets, The Journal of Fuzzy Mathematics 9 (3) (2001) 589 602.

15. P.K.Maji, R.Bismas, A.R.Roy, Soft set theory, Comput. Math. Appl. 45 (2003) 555-562.

16. P.K.Maji, A.R.Roy, An Application of soft sets in a decision making problem, Comput. Math. Appl.44 (2002) 1077-1083.

17. D. Molodtsov, Soft set theory-first results, Comput. Math. Appl.37 (1999), 19-31.

18. A.Rosenfeld, Fuzzy groups, Journal of Mathematical Analysis and Applications 35 (1971) 512-517. 
19. Smarandache, F., Neutrosophic set, a generalisation of the intuitionistic fuzzy sets, Int. J. Pure Appl. Math. 24, (2005) 287-297.

20. Qiu-Mei Sun, Zi-Liong Zhang, Jing Liu, Soft sets and soft modules, Lecture Notes in Comput. Sci. 5009 (2008) 403-409.

21. Zadeh L. A., Fuzzy Sets, Inform. Control 8, (1965) 338-353.

22. M.M. Zahedi, R.Ameri, On Fuzzy Projective and Injective Modules, The journal of Fuzzy Mathematics, Vol.3, No.1 (1995), 181-190. 